\title{
Short Pulse Propagation in an Inverted Two-Level Medium
}

\author{
G. G. Grigoryan ${ }^{a}$, V. O. Chaltykyan ${ }^{a}$, and V. S. Paturyan ${ }^{b}$ \\ ${ }^{a}$ Institute for Physical Research, NAS of Armenia, Ashtarak, Armenia \\ ${ }^{b}$ Computer Science Department, NUI Maynooth, Maynooth, Ireland \\ e-mail: gaygrig@gmail.com
}

Received May 17, 2011; in final form, June 1, 2011

\begin{abstract}
We consider propagation of a pulse carrying optical information in a resonant medium of two-level atoms and revisit the concept of the group velocity. We obtain conditions when this concept may be used and show that in a population inverted medium the possible superluminal propagation may result in advance times much shorter than the pulse duration because of lethargic amplification following from the complete exact solution of the problem.
\end{abstract}

Keywords: resonant medium, superluminal propagation, group velocity.

DOI: $10.3103 / \mathrm{S} 1060992 \mathrm{X} 11040035$

\section{INTRODUCTION}

Quantum informatics continues to be one of the very fast developing fields of scientific research. Successful experiments on storage and retrieval of optical information in different media [1-3] raised a new urgent problem that is transfer of undistorted optical information via resonant medium and creation of controllable delay or advance lines.

The extremely low group velocity achieved in experiments on slow light was $17 \mathrm{~m} / \mathrm{s}$ [4]. Considerable interest was also excited towards multiple experiments on the so called superluminal propagation in a medium (see, for example [5-10]) where an advance, as compared with vacuum, of the pulse peak value has been observed. Authors of these works interpret the obtained results as propagation of pulses at the velocity exceeding speed of light in vacuum and even at negative group velocity. Theoretical consideration in linear media [11-15] is based on the Fourier formalism. This formalism gives in the first approximation with respect to dispersion the well-known expression for the group velocity in a resonant medium,

$$
u=\frac{c}{n+\omega_{0} \frac{\partial n}{\partial \omega}},
$$

where $n(\omega)$ is the index of refraction of the medium and $\omega_{0}$ the pulse carrier frequency.

In the range of anomalous dispersion the derivative $\partial n / \partial \omega$ is negative and this may lead to not only the group velocity exceeding $c$, but to negative group velocity as well.

At first sight, expression (1) describes well the results observed in experiments mentioned above. However, the length of medium in all experiments is much shorter than the spatial size of the pulse and the observed group advance $T_{a d v}=x(1 / c-1 / u)$ is much shorter than the pulse duration. The questions whether the lengthening of medium would have lead to the increase in the group advance and how long is the longest possible $T_{a d v}$ remain thus unanswered. Moreover, the concept of the group velocity of an optical pulse in a resonant medium and the limits of applicability of this concept require more detailed study. As approximation of a medium without dispersion (inertia less), which in microscopic consideration corresponds to the approximation where the populations of atomic levels track the instantaneous value of the field, i.e., the adiabatic approximation, it may be assumed that the first order with respect to dispersion corresponds to the allowance for the first non-adiabatic correction.

The present work is an attempt to analyze the problem of superluminal propagation, in particular, from the point of view of causality principle; we choose for this purpose the simplest model where anomalous dispersion is possible, namely a medium of inverted two-level atoms. We will obtain the conditions of validity of the notion of group velocity and estimate the value of longest possible group advance. 


\section{SOLUTION OF PROPAGATION EQUATION}

Propagation of a short laser pulse in a resonant medium consisting of two-level atoms is described by the system of Maxwell and Schrödinger equations,

$$
\begin{aligned}
& \frac{\partial a_{1}}{\partial t}=i \Omega * a_{2}, \\
& \frac{\partial a_{2}}{\partial t}=i \Omega a_{1}-i \delta a_{2}, \\
& \frac{\partial \Omega}{\partial x}-\frac{1}{c} \frac{\partial \Omega}{\partial t}=i q a_{1}^{*} a_{2},
\end{aligned}
$$

where $\Omega$ is the Rabi frequency of the pulse, $a_{1,2}$ the amplitudes of the two atomic states, $\delta$ the detuning, and $q=2 \pi \omega d^{2} N / h c$. Note that we use here the Schrödinger equation with neglecting relaxations, hence this formalism is valid only in case of pulses whose duration is much shorter than all relaxation times. This will be the case under consideration in what follows.

By combining the equations above we can obtain the following equation of propagation (in running coordinates $x$ and $\tau=t-x / c$ ):

$$
\frac{\partial}{\partial x}\left(\frac{\partial \Omega}{\partial \tau}+i \delta \Omega\right)-q \Omega \sigma=0
$$

where $\sigma$ is the atomic level population difference, $\sigma=\left|a_{2}\right|^{2}-\left|a_{1}\right|^{2}$. In linear approximation $\sigma=1$ for the inverted medium and $\sigma=-1$ for the unexcited medium.

Equation (3) should be solved under the boundary and initial conditions $\Omega(\tau, x=0)=\Omega_{0}(\tau)$ and $\Omega(\tau \leftrightarrow \infty, x)=0$. The last condition follows from the initial condition imposed on the medium where all atoms should be in their initial state before the interaction with the pulse. In adiabatic approximation, i.e., neglecting the terms of the order $(T \delta)^{-1}$, Eq. (3) is reduced to $\partial \Omega / \partial x+i q \sigma \Omega / \delta=0$, whose solution is $\Omega(t, x)=\Omega_{0}(t-x / c) \exp (i q x \sigma / \delta)$. Thus, in adiabatic approximation the pulse envelope travels in the medium at the velocity $c$ and for the medium index of refraction the well-known expression $n=1+q \sigma / \delta$ is obtained and the pulse group velocity should equal

$$
u=c /\left(1-c q \sigma / \delta^{2}\right)
$$

in accordance with formula (1). So, the group velocity may be not only higher than $c$, but it may also be negative and even infinite depending on the value of the parameter $c q \sigma / \delta^{2}$.

Consider now the complete solution to Eq. (3), which satisfies the conditions above; it is given in the book [16]:

$$
\Omega(t, x)=\int_{-\infty}^{t}\left(\dot{\Omega}\left(t^{\prime}, 0\right)+i \delta \Omega\left(t^{\prime}, 0\right) e^{-i \delta\left(t-t^{\prime}\right)} I_{0}\left(2 \sqrt{q x\left(t-t^{\prime}\right.}\right) d t^{\prime} .\right.
$$

Here $I_{0}$ is the modified Bessel function. As follows from the analysis of this solution, which contains the entire dispersion series, the pulse suffers during propagation in medium the so called "lethargic amplification" [17-18] (not related with the relaxation constant $\Gamma$ ). At long times when the condition $\sqrt{q x(t-T)}>1$ is met, it follows from expression (5) that $|\Omega| \sim \operatorname{const}(\exp [2 \sqrt{q x t}])$.

Consider now the propagation length where the time of group advance may become of the order of the pulse duration. By substituting the value $T \delta^{2}$ instead of $q x$ into the exponent, one obtains an amplification of the order $\exp (2 \delta T)>>1$. With such an essential amplification, on the one hand, nonlinear effects will unavoidably be displayed and, on the other hand, it becomes meaningless to speak about the pulse propagation at a group velocity (i.e., without change in shape).

Figure 1 shows the spatial evolution (at different travelling lengths) of the temporal shape of the pulse envelope for different values of parameter $T \delta$. The plots are constructed from the formula (5). As seen in the figures, the pulse front moves at the velocity $c$ and the consequences of lethargic amplification are apparent. Sufficiently far from resonance (lower right figure) distortions of the pulse shape begin at the distance $q x T=4$. Closer to resonance (lower left figure) they begin at $q x T=2$, while at $q x T=4$ they are already considerable. Furthermore, at $q x T=6$ and $q x T=4$ (upper right plots) the temporal shape of the pulse has actually nothing common with the incident pulse shape. Finally, very close to resonance (upper left curves) pulse envelope changes dramatically at already $q x T=1$. (For example, for rubidium vapor with $N=10^{13} \mathrm{~cm}^{-3}$ we obtain $q=10^{10} \mathrm{CGS}$ and hence $q x T=1$ for nanosecond pulses corresponding to a length of about $0.1 \mathrm{~cm}$ ). 

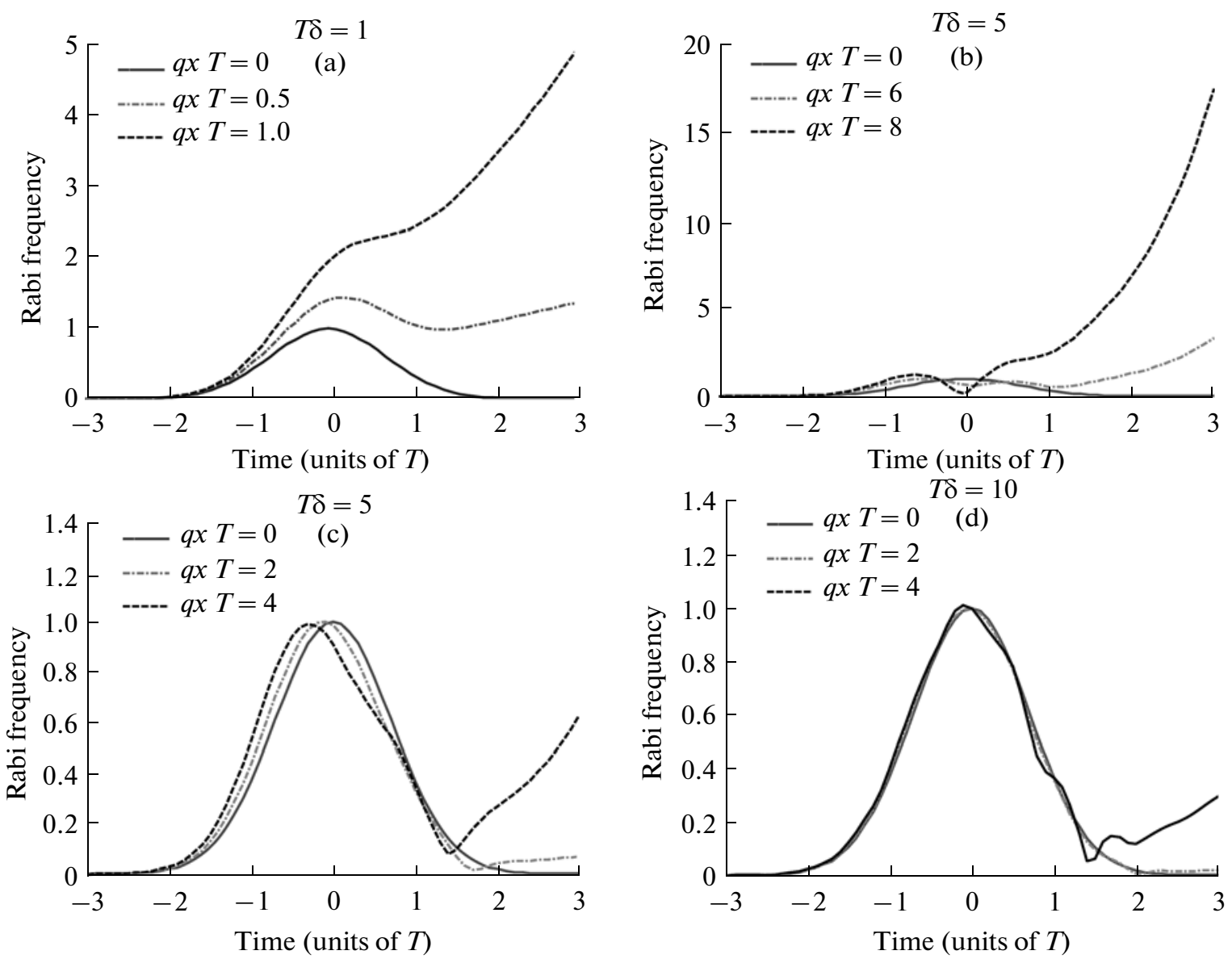

Fig. 1. Time evolution of the normalized Rabi frequency of the pulse for different propagation distances and different detunings.

\section{DISCUSSION}

The solution (5) is obtained by means of Laplace transformation with respect to $x$. But, as the equation (3) has constant coefficients, it allows also applying Fourier transformation with respect to time. This procedure results in the solution appearing usually in references:

$$
\Omega(\tau, x)=\int_{-\infty}^{+\infty} \Omega_{0}(\omega) e^{-\frac{i q x}{\omega+\delta}} e^{i \omega \tau} d \omega,
$$

We observe that this solution does not describe the lethargic amplification; the pulse energy is preserved during propagation in the medium $\left(|\Omega(\omega, x)|^{2}=\left|\Omega_{0}(\omega, x=0)\right|^{2}\right)$. This contradiction is explained by the fact that the expression (6), although being a solution to the Fourier-transformed equation, is not the solution to the considered problem with its initial conditions, because it does not go to zero when $t \leftrightarrow \infty$. Hence, only expression (5) should be considered as the correct solution. It should be noted that the expression (6) gives, in the first approximation with respect to dispersion, the same expression for the group velocity as above (4), but the condition of validity of the first dispersion approximation following from (6) is $T_{a d v} \ll T$ (where $T$ is the characteristic time of variation of the pulse envelope, which for smooth pulses may be defined as the pulse duration at half-maximum) and allows large group advance, e.g., exceeding the pulse duration even by an order of magnitude.

As the direct analysis of the exact solution (5) is rather cumbersome, let us go back to the equation (3). If we separate the rapidly oscillating phase $\exp (i q x / \delta)$ and pass to new variables $z=x$ and $\xi=\tau+q x / \delta^{2}$, we obtain for $\Omega_{1}(t, x)=\Omega(t-x / c) \exp (-i q x \sigma / \delta)$

$$
\frac{\partial}{\partial \xi}\left(\frac{\partial}{\partial z}-\frac{q \sigma}{\delta^{2}} \frac{\partial}{\partial \xi}\right) \Omega_{1}+i \delta \frac{\partial}{\partial z} \Omega_{1}=0
$$


Neglecting the terms of the order $q x \sigma / T \delta^{2}$, we have

$$
\frac{\partial}{\partial z}\left(\frac{\partial \Omega_{1}}{\partial \xi}+i \delta \Omega_{1}\right)=0
$$

Solution to this equation with the initial conditions above is $\Omega_{1}(\xi, z)=\Omega_{0}(\xi)=\Omega_{0}(t-x / u)$ with $u$ being the group velocity as defined in (3). The group advance (in units of $T$ ) is in this case $q x / T \delta^{2} \ll 1$. This approximate solution describes correctly the pulse motion within only time intervals satisfying the condition under which it was obtained, i.e., close to the pulse peak. At longer times $(|t|>T)$ the $q x / \delta^{2}$ must be omitted, so the pulse front travels at the speed $c$. If we rewrite the last condition as

$$
\left(q x / \delta^{2}\right)(x / c T)<<1,
$$

we observe that at high values of the first factor the notion of the group velocity exists for only the distances much shorter than the spatial size of the pulse. At larger distances the pulse energy suffers dramatic changes because of the lethargic amplification.

Note that lethargic amplification may also lead to violation of linear approximation, but this is out of scope of the present study.

\section{CONCLUSIONS}

Summarizing, we can state the following. The criterion of applicability of the concept of the group velocity is the inequality (9). At longer distance the pulse shape and hence the optical information it carries suffers essential distortion via lethargic amplification. The correct solution to the problem is in full agreement with the causality principle, and the possible group advance is always much shorter than the pulse duration.

\section{ACKNOWLEDGMENT}

Work was in part supported by the Ministry of Education and Science of Armenia (MESA), grant no. 11-1c124 of State Science Committee of MESA, IRMAS International Associated Laboratory, and the Volkswagen Stiftung.

\section{REFERENCES}

1. Fleischhauer, M., Imamoglu, A., and Marangos, J.P., Rev. Mod. Phys., 2005, vol. 77, p. 633.

2. Lukin, M.D., Rev. Mod. Phys., 2003, vol. 75, p. 457.

3. Heinze, G., Rudolf, A., Beil, F., and Halfmann, T., Phys. Rev. A, 2010, vol. 81, p. 001800.

4. Hau, L.V., Harris, S.E., Dutton, Z., and Behroozi, C.H., Nature, 1999, vol. 397, p. 594.

5. Wang, L.J., Kuzmich, A., and Dogariu, A., Nature, 2000, vol. 406, p. 277.

6. Bigelow, M.S., Lepeshkin, N.N., and Boyd, R.W., Science, 2003, vol. 301, p. 200.

7. Stenner, M.D., Gauthier, D., and Neifeld, M., Nature, 2003, vol. 425, p. 695.

8. Michajlov, E., Sautenkov, V., Novikova, I., and Welch, G.R., Phys. Rev. A, 2004, vol. 69, p. 063808.

9. Lezam, A., Akulshin, A.M., Sidorov, A.I., and Hannaford, P., Phys. Rev. A, 2006, vol. 73, p. 033806.

10. Tanaka, H., Niwa, H., Hayami, K., Furue, S., Nakayama, K., Kohmoto, T., Kunitomo, M., and Fukuda, Y., Phys. Rev. A, 2003, vol. 68, p. 035801.

11. Garrett, C.G.B. and McCumber, D.E., Phys. Rev. A, 1970, vol. 1, p. 305.

12. Chiao, R.Y., Phys.RevA, 1993, vol. 48, p. 34.

13. Payne, M.G. and Deng, L., Phys. Rev. A, 2001, vol. 64, p. 031802.

14. Ghulghazaryan, R.G. and Malakyan, Y.P., Phys. Rev. A, 2003, vol. 67, p. 063806.

15. Dogariu, A., Kuzmich, A., Cao, H., and Wang, L.J., Optics Express, 2001, vol. 8, p. 344.

16. Mikaelian, A.L., Ter-Mikaelian, M.L., and Turkov, Yu.G., Opticheskiye generatory na tverdom tele (Solid-State Optical Generators), Moscow: Sovetskoye Radio, 1967.

17. Chung, H.K., Lee, J.B., and DeTemple, T.A., Opt. Com., 1981, vol. 39, p. 105.

18. Kryzhanovskii, B.V., Opt. i Spektrosk., 1989, vol. 67, p. 309. 